\title{
Domain of impact for agents collaborating in a baggage handling system ${ }^{\star}$
}

\author{
Kasper Hallenborg \\ Maersk Mc-Kinney Moller Institute \\ University of Southern Denmark \\ Odense M, 5230, Denmark \\ hallenborgemmmi.sdu. dk
}

\begin{abstract}
Recognize the frustration of waiting in slow starting queues? Acceleration ramps and reaction times make traffic queues frustrating to many people every day. Similar problems arise in many production and material handling systems.

In this paper we present research activities conducted on a baggage handling system (BHS) of a large airport hub in Asia, where we have applied an intelligent multi-agent based approach to control the flow of bags on the BHS. By exchanging a centralized control system with an agent-based solution, local queues can be avoided or minimized, which increase the overall performance of the BHS.

Through an established community of highly collaborating and coordinating agents, each agent can, based on its relative placement in the topology of the BHS, decide whether it is appropriate to route more bags through this node relative to the overall system load. The agentbased approach not only improves robustness of the system, and utilize the entire BHS in a more convenient and dynamical way, it also include strategies for maximizing capacity of the system.

We present results from ongoing work of developing suitable and proficient algorithms and agent collaboration schemes to increase the performance of the BHS. In this paper we pay special attention to the impact of the relative physical displacement of the agents in the system.
\end{abstract}

Key words: Multi-agent systems, decision logic, production systems, graphs

\subsection{Introduction}

For historic reasons (The Denver Airport software scandal) the Airport industry has been rather conservative about introducing new approaches and intelligent control in baggage handling systems.

A baggage handling system (BHS) transfers baggage in major airports between the arrival and departure gates, and from early check-ins. In setup and

* This research was supported by The Ministry of Science, Technology and Innovation in Denmark under the IT Cooridor Foundation

Please use the following format when citing this chapter:

Hallenborg, K., 2007, in IFIP International Federation for Information Processing, Volume 247, Artificial Intelligence and Innovations 2007: From Theory to Applications, eds. Boukis, C., Pnevmatikakis, L., Polymenakos, L., (Boston: Springer), pp. 243-250. 
functionality a BHS is comparable to many manufacturing systems - bags enter the system through various channels, undergo various processing (mainly routing), before it leaves the BHS at the departure gates.

A BHS is a huge mechanical system, usually composed of conveyor-like modules capable of transferring totes (plastic barrels) carrying one bag each $^{2}$. We have researched a BHS for a major airport hub in Asia, with more than 5000 modular components each with a length of 2-9 $\mathrm{m}$ running at speeds from $2-$ $7 \mathrm{~m} / \mathrm{s}$. The BHS alone can easily be up to $20 \mathrm{~km}$. in total length and may cover an area of up to $600.000 \mathrm{~m}^{2}$. A BHS should be capable of handling more than 100.000 pieces of baggage every day, and for the researched airport the maximum allowed transfer time is $8-11$ minutes for a distance of up to $2.5 \mathrm{~km}$.

\subsubsection{Main functionality}

The core task of a BHS is transferring bags from $A$ to $B$, but a highly dynamic environment complicates the control and optimization of capacity in the system. Changes in flight schedules, lost baggage information, and breakdowns are factors, which in combination with peak loads on the system result in queues and delayed baggage. Dischargers at the departure gates are temporarily allocated to one or more flights. Totes carrying bags are discharged (unloaded) when they reach the correct discharger according to their flight destination. Identity and destination of the bags is unknown until scanned at the input facility. After discharge the empty tote continues on the BHS back to the input facility sharing the conveyor lanes with other full totes. Thus routing of empty totes clearly impact the performance of the BHS. As no reliable model exists for arrival of bags to the system, and given the complexity of the BHS and time constraints of travelling bags (5-12 min as allowed max. transfer time) makes exact off-line scheduling impossible. Because totes or DCVs in many systems have to stop or slow down when discharging or unloading, respectively, the capacity for that lane section goes down, and a queue can accumulate behind the discharger, therefore more dischargers are often allocated to the same flight in order to distribute the load on the entire BHS. Traditionally the control software of the BHS is built on a simple reliable centralized approach based on static shortest paths of the system. Each pre-calculated route between toploaders and dischargers are given a route number and when the destination of a bag is known by the system, it follows that route until it reaches the destination.

Thus the structure, complexity and task make it an appropriate candidate for a decentralized agent-based control system with local observations.

\subsubsection{MAS technology}

MAS technology, which spawned from artificial intelligence as DAI (Distributed Artificial Intelligence) [?], offers an approach to decompose complexity of systems into a number of collaborating autonomous agents. System-wide tasks are

\footnotetext{
${ }^{2}$ Some BHSs are based on AGV-like telecars, which autonomously run on the BHS
} 
solved partly by subtasks in the individual agents, which are coordinated and aligned through their interaction patterns. Interaction schemes and communication protocols for agents can be specified or programmed in an ad-hoc or domain specific manner, but to increase common understanding and platform independence, FIPA provides a set of specifications for interaction protocols supporting both negotiation and co-ordination between agents [?].

\subsubsection{DECIDE project}

Our research case of the BHS is conducted in collaboration with the company installing and producing the BHS, FKI Logistex. This case is part of a larger research project called DECIDE, which focus on promoting and proving the appropriateness of multi-agent based control in production and manufacturing systems. Major Danish manufactures are among the other partners of the consortium: Lego, Grundfos, Bang and Olufsen (B\&O), and Odense Steel Shipyard.

\subsection{System setup}

Recent years advancement in computer performance has made it possible to do realistic real-time simulations of very complex environments. The ability to continuously interact with the simulation model during operation creates a perfect off-site test-suite for the control-software, which emulates the real BHS.

Together with another consortium partner, Simcon, the BHS company FKI Logistex has created an emulation model of the researched BHS using the AutoMod simulation and modeling package $[?]^{3}$. One of the strong advantages of using AutoMod is concurrent communication with the model identical to the connection between the control server and the PLCs in the real hardware. Thus the control software cannot see the difference, if it is connected to the emulation model or the real hardware. A snapshot of the emulation model is shown in the figure 1.1. It shows the area with input facilities for terminal 3 of the airport.

\subsubsection{Modeling with agents}

In abstract form the BHS can be understood as a directed graph of connected nodes, which represent elements of the real BHS, where it is possible to load, unload, or redirect the totes (toploading, discharging, merging, and diverting elements). Given the layout of the BHS no practical arguments exists for making decisions for a tote between nodes of the graphs. Thus an intuitive approach to decompose the decision logic to a multi-agent system is to place agents in each node corresponding to diverters, mergers, toploaders, dischargers, etc.

\footnotetext{
${ }^{3}$ AutoMod is a de-facto standard for systems analysis of manufacturing and material handling systems.
} 


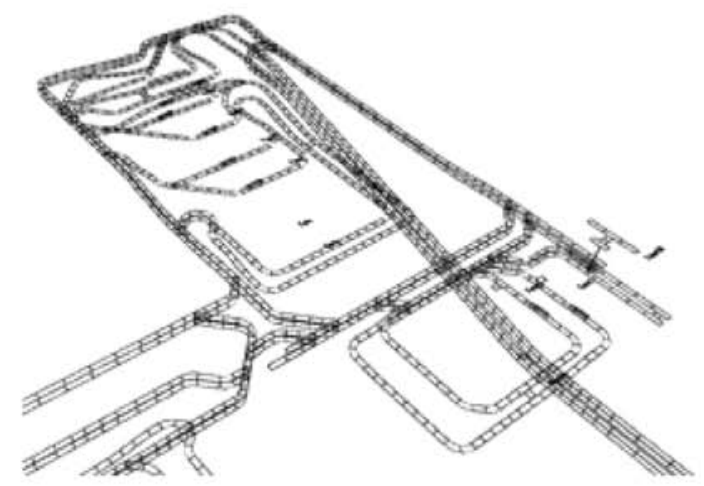

Fig. 1.1. Snapshot of the input area of the investigated BHS

If the design of the agents is simple and intuitive, the modeling of the associations and collaborations between the agents are far more complex. Ideally, with respect to generality and simplicity in the design of the agents and control software, the domain of observation for each agent should only span the edges from the agent's node to the next node in the graph (input and output lanes of conveyors at each node). But for some decisions, agents require more information about the current status of the load on the entire system, e.g. when deciding between alternative dischargers far from the current location.

Forced by both economical and architectural constraints of the airport, the layout of the BHS would usually have a rather low density of lanes and alternative routes compared to communication networks or traffic systems. The low density of connections in the graphs and the limited number of alternatives routes, makes the BHS less appropriate for intelligent network routing algorithms, such as ant-based control by Schoonderwoerd et al. [?] or AntNet by Di Caro and Dorigo [?]. Another important difference between communication strategies and the flow of bags in the BHS, is that a package can always be resubmitted in a package-switched network, that is not an option in the design of a BHS. In contrast to traffic control systems the BHS is actual aware of the correct destination for a tote, as soon as the bags enters the BHS, which makes it more attractive to use more system-wide collaboration of the agents. This could be achieved by assisting the local node agents with a number of mediator agents, which can be queried by local agents about information of routes through the entire system in order to discover, which agents to collaborate and negotiate with. Mediator agents can become a bottleneck or single-point-of-failure points of the system. Another approach is to profile each agent with some relevant knowledge of the topology of the BHS. We have successfully worked with both approaches with no significant difference, besides a lager communication overhead in a mediator based solution. 
The important aspect is not how an agent discovers other agents to collaborate and negotiate with in order to route a current tote correctly, but which impact these different agents should have on the decision in the local agent.

As all destinations somehow can be reached from anywhere in the system, one could argue that the status and load of every node in the system should be considered at every decision point, but then complexity increases exponentially, and is both practical impossible and inappropriate for performance reasons. For capacity and space utilization reasons a BHS is often built with 2 or more layers of conveyors vertically displaced. Dischargers placed above each other are usually allocated to the same flight, because an even distribution of totes to two dischargers copes with the lower capacity of dischargers compared to traditional lane elements (discharging is 2 times slower than average lane speed). Due to cost there is only few locations (2-3), where you can go from one layer to another. Thus making the right decision at these points is far more important than minor redirection on the same layer - similar to the importance of taking the right exit on a highway compared to turns in a denser road system of a city.

In the following section we will present different strategies used for different type of decisions in the BHS, to illustrate the importance of considering the domain of impact on the agent's decision logic.

\subsection{Agent strategies}

The primary reason for exchanging the conventional control software with an agent-based approach is to decrease complexity and minimize dependencies in the control logic of the BHS. A multi-agent solution allows more advanced strategies to be used because the control logic of each node is simple, and altogether both capacity and robustness should be increased.

The basic building block in the strategies of the agents are simple observations of the local neighborhood of each agent - status or queue observations. Each node agent in the BHS collects information about the status of its local domain, which means the conveyor lanes to the previous and the following nodes in the graph. The information collected expose values of a edge/lane, such as the number of totes per element, the average delay for totes, and the average urgency of bags, which means how close a bag is to its departure (in time).

\subsubsection{Overtaking urgent bags}

An example of a collaborating strategy, which has a little impact on the domain of neighboring mergers and diverters, allows urgent bags to overtake non-urgent bags, by detouring non-urgent bags. Consider a typical layout of a discharging area in figure 1.2. The bottom lane is a fast forward transport line, the middle a slower lane with the dischargers and the upper lane is the return path. A diverter (in the bottom lane) has the option to detour non-urgent to the middle lane to give way for urgent baggage in the transport line. If there is no load 
on the system all totes follows the shortest path. When the routes merge again at the mergers in the middle lane, it will give higher priority to totes from the merging leg with the most urgent baggage.

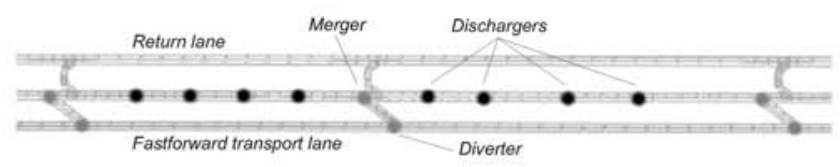

Fig. 1.2. Snapshot of the BHS layout with indication of diverters, mergers, and dischargers

The urgency observation in a node agent is composed of two custom fuzzy sets created from standard hedges [?], which are biased towards a desired neutral point of urgency. Then urgency of a bag goes exponentially up as it approaches departure, and turns negative if it has more than $20 \mathrm{~min}$. to departure. Thus likelihood of detouring is directly given from the urgency information.

The strategy presented above allows urgent baggage to overtake baggage that have plenty of time to departure, is an example of a strategy, where the domain of impact only range between two succeeding nodes of the graph. Experiments showed impressive results without extending the domain of impact.

The strategy for returning empty totes to tote stackers, which are located close to the toploaders, is an example of collaboration between agents, where the domain is much broader and many agents participate. The full status of each tote stacker is, similar to the urgency status of the nodes, composed of fuzzy sets, which secures that the request for empty totes increases exponentially, when the tote stacker is almost empty. Besides the status of the tote stacker also the distance to the tote stacker should be considered, it gives no sense to route an empty tote to the other end of the BHS, if another tote stacker is very close, unless the far one is almost empty. Also the load on the node agents along the route to the tote stackers could influence the decision, as it would be preferred to send empty totes along a route, where they do not obstruct the way for full totes. The load status of the nodes is therefore taken into account as weight in the choice of the destination for the empty tote, similar to how the load status of nodes agents are considers in the strategy for saturation management presented in the next section.

\subsubsection{Saturation management and the WIPAC curve}

Saturation management is a strategy with the purpose of avoiding queues at all by minimizing the load on the system in critical areas. The issues on acceleration ramps and reaction times mentioned in the beginning result in the characteristics of the BHS known as the work in-progress against capacity curve (WIPAC), which is further described in [?]. In principle it states that the capacity of the systems goes dramatically down, if the load on the system exceeds a 
certain threshold value - the system more or less end up in a deadlock situation where everything get stocked. The curve is dynamical, due to the various load on the system, and maximum cannot be calculated exactly. Thus the strategy is to quickly respond to minor observations, which indicates that the maximum has been reached, and then block inputs to the area. The strategy is simply to block a toploader if routes from the toploader are overloaded and let the system resolve. Queues close to the toploader are most critical, as the toploader has great impact on filling up those queues, whereas parts of the route far from the toploader could have been resolved before the new totes arrive. Instead of blocking the toploader, we can just slow down the release of new totes using the following fraction of full speed for the toploader.

$$
v_{\tau} \frac{\sum_{i} w_{i} q_{i}}{w_{i}}=v_{\tau} \frac{\sum_{i} \frac{\alpha}{d_{i}} q_{i}}{\sum_{i} \frac{\alpha}{d_{i}}}
$$

where $v_{\tau}$ is the full speed of the toploader, and $w_{i}$ are weights of the queue statues, $q_{i}$, along the routes. The weight is given by a coefficient $\alpha$ and the distance from the toploader $d_{i}$. Queue status is a number between 0 and 1 , where 1 indicates no queue. The effect of the saturation management strategy is clearly documented by the graph in figure 1.3, and the domain of impact by other agents is almost system-wide for this strategy as well.

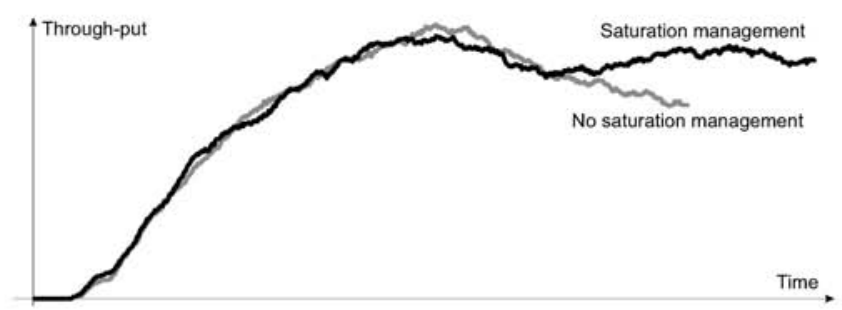

Fig. 1.3. Result of a test scenario with and without saturation management

\subsection{Conclusion and future work}

Is this paper we have presented important research contributions from the DECIDE project about multi-agent based control of a baggage handling system (BHS) in a major airport hub in Asia. The agent-based approach has spread the decision and control logic of the system to a large number of collaborating agents and replaced a complex centralized control structure. It has enabled new strategies and observations in the local agents to increase robustness, capacity, and throughput of the BHS. Special attention has been given to the domain of impact on the decision logic and collaboration among the agents, which varies depending on the strategy. 
We continue our research on the BHS and will develop more new strategies for the agents, and increase their mutual collaboration to maximize the utilization of the BHS during peak times. We will try to avoid the use of mediator agents and rely on roles and profiles. Ideally a swarm of local agents would provide a general setup that easily can be ported to other systems. During the research we will pay special attention to develop abstract and general design methodologies for the topological domain of impact for agent collaborations.

\section{References}

1. Weiss, G., ed.: Multiagent Systems - A modern approach to distributed artifical intelligence. MIT Press (1999)

2. : FIPA - The Foundation of Intelligent Physical Agents. http://www. fipa.org/ (2006)

3. Brooks Software: Automod - simulation and modeling software. http://www . brookssoftware.com (2006)

4. Schoonderwoerd, R., Holland, O., Bruten, J., Rothkrantz, L.: Ant-based load balancing in telecommunication networks. Adaptive Behaviour (5) (1996) 169-207

5. Caro, G.D., Dorigo, M.: Antnet: A mobile angents approach to adaptive routing. Technical Report IRIDIA/97-12, Université Libre de Bruxelles, Belgium (1997)

6. Negnevitsky, M.: Artificial Intelligence A Guide to Intelligent Systems. 2 edn. Addison Wesley (2005)

7. Kragh, A.: K-Netvrksmodeller til Analyse af FMS Anlg. PhD thesis, Informatics and Mathematical Modelling, Technical University of Denmark (1990) 